TRANSACTIONS OF THE

AMERICAN MATHEMATICAL SOCIETY

Volume 359, Number 10, October 2007, Pages 4757-4771

S 0002-9947(07)04047-0

Article electronically published on April 24, 2007

\title{
EMBEDDABILITY OF SOME STRONGLY PSEUDOCONVEX CR MANIFOLDS
}

\author{
GEORGE MARINESCU AND NADER YEGANEFAR
}

\begin{abstract}
We obtain an embedding theorem for compact strongly pseudoconvex CR manifolds which are boundaries of some complete Hermitian manifolds. We use this to compactify some negatively curved Kähler manifolds with compact strongly pseudoconvex boundary. An embedding theorem for Sasakian manifolds is also derived.
\end{abstract}

\section{INTRODUCTION}

A natural question in the study of $\mathrm{CR}$ manifolds is the embeddability question: given a strongly pseudoconvex CR manifold $X$, do there exist an integer $N$ and a smooth embedding $M \hookrightarrow \mathbb{C}^{N}$ such that the CR structure on $X$ is the one induced from the complex structure on $\mathbb{C}^{N}$ ? It is known that the embedability of $X$ is equivalent to the fillability, that is, the existence of a compact complex manifold $T$ with strongly pseudoconvex boundary, such that $\partial T=X$. Indeed, HarveyLawson's theorem [HL75] shows that $X$ is fillable if it is embeddable. Conversely, if $X$ is fillable and $\partial T=X$, a theorem of Grauert Gra62 shows that $T$ can be taken to be a Stein space with isolated singularities, and one can apply the embedding theorem of Remmert-Bishop-Narasimhan [Nar60.

By a theorem due to Boutet de Monvel BdM75], any compact (2n-1)-dimensional strongly pseudoconvex $\mathrm{CR}$ manifold is embeddable in some $\mathbb{C}^{N}$ if its dimension is greater than or equal to five $(n \geqslant 3)$. On the other hand, there is a classical example of H. Rossi [Ros65] (see also [Bur79, Fal92]) which shows that an arbitrarily small, real-analytic perturbation of the standard structure on the three-sphere may fail to be embeddable. There exists an extensive literature on the embeddability of deformations of CR structures; see e.g. J. Bland and C. L. Epstein BE96, D. M. Burns and C. L. Epstein [BE90, C. L. Epstein and G. M. Henkin [EH00, L. Lempert Lem95.

Our goal is to get embedding theorems for compact strongly pseudoconvex CR manifolds, which are already boundaries of non-compact strongly pseudoconvex complex manifolds (see Definition 3.1). The complex manifolds we consider are complete Hermitian manifolds with an additional assumption on the spectral prop-

Received by the editors January 10, 2005 and, in revised form, April 18, 2005.

2000 Mathematics Subject Classification. Primary 32V30, 32V15, 32Q05.

The second-named author was (partially) supported by the European Commission through the Research Training Network HPRN-CT-1999-00118 "Geometric Analysis". 
erties of the operator $\bar{\partial}+\bar{\partial}^{*}$ acting on square integrable forms. Our main technical result is the following theorem:

Theorem 1.1. Let $M$ be a complex manifold with compact strongly pseudoconvex boundary and of complex dimension $n \geqslant 2$. Assume that $\operatorname{Int} M$ has a complete Hermitian metric such that $\left(\bar{\partial}+\bar{\partial}^{*}\right)^{2}$ is Fredholm in bidegree $(0,1)$. Then there exists a $C R$ embedding of the boundary $\partial M$ in $\mathbb{C}^{N}$ for some integer $N$.

We then give two applications of this result. The first one shows that manifolds with pinched negative curvature, compact strongly pseudoconvex boundary and finite volume (away from a neighborhood of the boundary) can be compactified to a strongly pseudoconvex domain in a projective variety by adding an exceptional analytic set, which can be blown down to a finite set of singular points.

Theorem 1.2. Let $M$ be a connected complex manifold with compact strongly pseudoconvex boundary and of complex dimension $n \geqslant 2$. Assume that Int $M$ is endowed with a complete Kähler metric with pinched negative curvature, such that away from a neighborhood of $\partial M$, the volume of $M$ is finite. Then

(1) $\partial M$ is embeddable in some $\mathbb{C}^{N}$.

(2) There exists a compact strongly pseudoconvex domain $D$ in a smooth projective variety and an embedding $h: M \rightarrow D$ which is a biholomorphism between $\operatorname{Int} M$ and $h(\operatorname{Int} M), h(\partial M)=\partial D$, and $D \backslash h(M)$ is an exceptional analytic set which can be blown down to a finite set of singular points.

It follows from the proof that each end of $M$, except that corresponding to $\partial M$, can be analytically compactified by adding one singular point of the Remmert reduction of $D$. There exists a bijective correspondence between the singular set of the Remmert reduction of $D$ and the ends of $M$ different from the end corresponding to $\partial M$. Theorem 1.2 is a generalization of [SY82, Main Theorem] in the case when a strongly pseudoconvex end is allowed. The method follows NR98 and consists in filling $\partial M$ with a Stein space $S$ (this is granted by Theorem 1.1) and using a theorem of Lempert Lem95, Theorem 1.2] to embed $S$ in a projective variety $X$. The complement $Y:=X \backslash S$ can be glued to the original manifold $M$ to construct a projective pseudoconcave manifold $\widehat{M}$. By using the results of And63 we can compactify this manifold, and by SY82] or MD06] the complement of $\widehat{M}$ is an exceptional analytic set.

As a consequence we study some quotients of the unit complex ball $B$ in $\mathbb{C}^{n}$ which were considered by Burns and Napier-Ramachandran [NR98, Theorem 4.2].

Corollary 1.3. Let $\Gamma$ be a torsion-free discrete group of automorphisms of the unit ball $B$ in $\mathbb{C}^{2}$ and let $M=B / \Gamma$. Assume that the limit set $\Lambda$ is a proper subset of $\partial B$ and that the quotient $(\partial B \backslash \Lambda) / \Gamma$ has a compact component $A$. Let $E$ be the end of $M$ corresponding to $A$ and assume that $M \backslash E$ has finite volume. Then $A$ is embeddable in some $\mathbb{C}^{N}$ and $M$ can be compactified to a strongly pseudoconvex domain in a projective variety by adding an exceptional analytic set.

In NR98 the case $n \geqslant 3$ is considered, and it is shown that the volume of $M \backslash E$ is automatically finite. Indeed, if $n \geqslant 3, \partial M$ is embeddable by [BdM75] and the method described above, combined with the holomorphic Morse inequalities of [NT88], shows that $\operatorname{vol}(M \backslash E)<\infty$. Napier and Ramachandran also remark [NR98, Remark, p. 392] that one could prove an analogue of Corollary 1.3 in the case $n \geqslant 3$, by using the techniques of Siu-Yau SY82]. 
Our second application of Theorem 1.1] is an embedding result for Sasakian manifolds (the definition of this particular class of strongly pseudoconvex manifolds will be given in $\S 5$ ). Let $X$ be a Sasakian manifold. In [BH02, O. Biquard and M. Herzlich construct a Kähler metric on the product $(0, \infty) \times X$, called an asymptotically complex hyperbolic metric. $X$ can then be viewed as the pseudoconvex boundary of some Kähler manifold, and therefore our technique can be applied to prove

Theorem 1.4. Let $X$ be a Sasakian manifold of dimension at least 3 . Then there is a $C R$ embedding of $X$ in $\mathbb{C}^{N}$ for some integer $N$.

This paper is organized as follows. In $\S 2$, we disscuss the Hodge decomposition for the space of square integrable forms. In $\S 3-4$, we prove Theorems 1.1 and 1.2 Finally in $\S 5$, we review some basic facts about Sasakian manifolds, explain the construction of asymptotically hyperbolic manifolds, and prove Theorem 1.4 .

\section{Hodge DECOMPOSITION}

Let $(M, g)$ be a complex complete Hermitian manifold. For any integers $p$ and $q$, we denote by $C_{0}^{p, q}(M)$ the space of smooth $(p, q)$-differential forms with compact support, and by $L_{2}^{p, q}(M)$ the space of square integrable $(p, q)$-forms. Now consider the operator $\bar{\partial}+\bar{\partial}^{*}$, where $\bar{\partial}$ acts as an unbounded operator on square integrable forms, and $\bar{\partial}^{*}$ is the Hilbert space adjoint of $\bar{\partial}$. The $L_{2}$-kernel $\mathscr{H}^{p, q}(M, g)$ of $\bar{\partial}+\bar{\partial}^{*}$ is by definition the space of $L_{2}$ harmonic $(p, q)$-forms. In order to solve the $\bar{\partial}$-equation, one is primarily interested in knowing whether $\mathscr{H}^{p, q}(M, g)$ is finite dimensional and $\bar{\partial}$ has a closed range as an unbounded operator acting on $L_{2}$-forms. For example, this is the case if $\bar{\partial}+\bar{\partial}^{*}$ (or equivalently $\bar{\square}:=\left(\bar{\partial}+\bar{\partial}^{*}\right)^{2}$ ) is Fredholm on its domain. It is well known that we have the following characterization of Fredholmness (see for example Ang93).

Proposition 2.1. The operator $\left(\bar{\partial}+\bar{\partial}^{*}\right)^{2}$ is Fredholm in bidegree $(p, q)$ if and only if we have a Poincaré inequality at infinity: there exist a compact subset $K$ of $M$ and a constant $C>0$ such that

$$
\forall u \in C_{0}^{p, q}(M \backslash K), C\|u\|_{L_{2}}^{2} \leqslant\|\bar{\partial} u\|_{L_{2}}^{2}+\left\|\bar{\partial}^{*} u\right\|_{L_{2}}^{2} .
$$

Our discussion applies to weighted spaces too. Namely, let $\xi: M \rightarrow \mathbb{R}$ be a smooth function defined on $M$, and let $L_{2, \xi}(M)$ be the Hilbert space completion of $C_{0}^{*, *}(M)$ with respect to the weighted norm

$$
\|u\|_{L_{2, \xi}}^{2}=\int_{M}|u|^{2} e^{-\xi} \mathrm{dv}_{\mathrm{g}}
$$

where $\mathrm{dv}_{\mathrm{g}}$ is the Riemannian measure. The operator $\bar{\partial}$ acts as an unbounded operator on $L_{2, \xi}(M)$, and its adjoint is denoted by $\bar{\partial}_{\xi}^{*}$; we have

$$
\bar{\partial}_{\xi}^{*}=e^{\xi} \bar{\partial}^{*} e^{-\xi} .
$$

The space of $\left(\bar{\partial}+\bar{\partial}_{\xi}^{*}\right)$-harmonic $(p, q)$ forms is

$$
\mathscr{H}_{\xi}^{p, q}(M, g)=\left\{u \in L_{2, \xi}^{p, q}(M): \bar{\partial} u=\bar{\partial}_{\xi}^{*} u=0\right\} .
$$


A key property of Fredholmness is the following:

Proposition 2.2. Let $(M, g)$ be a complete Hermitian complex manifold. Assume that $\bar{\partial}+\bar{\partial}_{\xi}^{*}$ is Fredholm in bidegree $(p, q)$. Then $\mathscr{H}_{\xi}^{p, q}(M, g)$ is finite dimensional, and we have the following strong orthogonal Hodge decomposition:

$$
L_{2, \xi}^{p, q}=\mathscr{H}_{\xi}^{p, q}(M, g) \oplus \operatorname{Im}\left(\bar{\partial}: L_{2, \xi}^{p, q-1} \rightarrow L_{2, \xi}^{p, q}\right) \oplus \operatorname{Im}\left(\bar{\partial}_{\xi}^{*}: L_{2, \xi}^{p, q+1} \rightarrow L_{2, \xi}^{p, q}\right) .
$$

For the proof of Theorem 1.2 we need the following example of manifolds for which the operator $\left(\bar{\partial}+\bar{\partial}^{*}\right)^{2}$ is Fredholm at certain degrees.

Proposition 2.3 (Gromov). If $(M, g)$ is a complete Kähler manifold whose fundamental form is d(bounded) (at least outside a compact subset) in the sense of Gromov, the operator $\left(\bar{\partial}+\bar{\partial}^{*}\right)^{2}$ is Fredholm in bidegree $(p, q)$, if $p+q \neq \operatorname{dim}_{\mathbb{C}}(M)$.

Recall that the hypothesis means that for a bounded 1 -form $\eta$, the fundamental form $\omega$ of the metric $g$ satisfies $\omega=d \eta$ outside a compact subset $K$. Then the method of proof of Gro91, Theorem 1.4.A], using the Lefschetz decomposition theorem, shows that for some constant $C>0$ depending only on the dimension of $M$ and the bound on the norm of $\eta$, we have

$$
\forall p+q \neq \operatorname{dim}_{\mathbb{C}}(M), \forall u \in C_{0}^{p, q}(M \backslash K), C\|u\|_{L_{2}}^{2} \leqslant\langle\Delta u, u\rangle_{L_{2}}=2\langle\bar{\square} u, u\rangle_{L_{2}} .
$$

Here, the last equality is a consequence of the Kähler assumption, for we then have $\Delta=2 \bar{\square}$, and after integrating by parts, we get the estimates of (2.1).

\section{EMBEDding OF SOME STRONGLY PSEUdoCONVEX CR MANIFOLDS}

The aim of this section is to prove Theorem 1.1. We first review the notion of a CR structure and a complex manifold with strongly pseudoconvex boundary. In Lemma 3.3, we construct a complete metric and a suitable weight $\xi$ on a strongly pseudoconvex subdomain $\Omega_{0}$ of $M$, such that the operator $\left(\bar{\partial}+\bar{\partial}_{\xi}^{*}\right)^{2}$ is Fredholm in bidegree $(0,1)$. Then Lemma 3.4 provides peak holomorphic functions at $\partial \Omega_{0}$. We use these peak functions in the proof of Theorem 1.1 in order to fill $\partial \Omega_{0}$ and then $\partial M$.

Let $\Omega$ be a strongly pseudoconvex domain in a complex manifold $M$. From the complex structure of $M$, we can build on the boundary of $\Omega$ a partial complex structure which is called a Cauchy-Riemann or CR structure. For this notion we refer to [BdM75, BE90, Bur79] and Section 5. We also need the notion of a complex manifold with strongly pseudoconvex boundary.

Definition 3.1. A complex manifold $M$ with strongly pseudoconvex boundary is a real manifold with boundary, of dimension $2 n$, satisfying the following conditions: (i) the interior Int $M=M \backslash \partial M$ has an integrable complex structure and (ii) for each point $x \in \partial M$ there exist a neighborhood $U$ in $M$, a strongly pseudoconvex domain $D \subset \mathbb{C}^{n}$ with smooth boundary, and a diffeomorphism $h$ from $U$ onto a relatively open subset $h(U)$ such that $h(\partial U) \subset \partial D$ and $h$ is biholomorphic from Int $U$ to $\operatorname{Int} h(U)$.

Actually, by the Newlander-Nirenberg theorem for manifolds with boundary of Catlin Cat the conditions of the definition are fulfilled if the manifold $M$ possesses 
an integrable complex structure, smooth up to the boundary, and for which the boundary is strongly pseudoconvex. From this definition we infer:

Consequence 3.2. The complex structure induces an integrable Cauchy-Riemann structure on the boundary $\partial M$. Moreover, if $\partial M$ is compact, there exists a defining function $\varphi: M \rightarrow(-\infty, c]$ such that $\partial M=\{\varphi=c\}$, with the properties: (1) its Levi form is positive definite on the holomorphic tangent space of $\partial M$ and (2) $\varphi$ is strictly plurisubharmonic on $\left\{c_{0}<\varphi<c\right\}$. We can assume that $c_{0}<0<c$ and that 0 is a regular value of $\varphi$, and consider the strongly pseudoconvex domain $\Omega_{0}=\{\varphi<0\}$.

For the lemmas leading to Theorem [1.1, we construct a smooth function $\lambda$ : $(-\infty, 0) \rightarrow \mathbb{R}_{+}$such that $\lambda=0$ in a neighborhood of $-\infty$ and $\lambda^{\prime}>0, \lambda^{\prime \prime}>0$ in a neighborhood of 0 . We will impose two conditions on the growth of $\lambda$ near 0 . Let $0<\delta<C$ be two constants and let $\psi: \Omega_{0} \rightarrow \mathbb{R}$ be a smooth function such that

$$
\psi= \begin{cases}-(n+2) \log (-\varphi) & \text { on }\{\varphi>-\delta\} \\ 0 & \text { on }\{\varphi<-C\} .\end{cases}
$$

First, we may choose $\lambda$ such that

$$
\lambda(\varphi) \geqslant \psi \text { on } \Omega_{0} .
$$

Lemma 3.3. Assume that $\operatorname{Int} M$ can be endowed with a complete Hermitian metric such that the operator $\left(\bar{\partial}+\bar{\partial}^{*}\right)^{2}$ is Fredholm in bidegree $(0,1)$. Then there exists on $\Omega_{0}$ a complete Hermitian metric (sending the boundary at infinity) for which the weight $e^{-\lambda(\varphi)}$ is integrable near the boundary and the operator $\left(\bar{\partial}+\bar{\partial}_{\lambda(\varphi)}^{*}\right)^{2}$ is Fredholm in bidegree $(0,1)$.

Proof. Consider the complete metric $g$ on $\Omega_{0}$ obtained by gluing the original metric of $M$ with the Kähler metric defined near the boundary $\partial \Omega_{0}$ by the Kähler form

$$
\begin{aligned}
\omega & =-\sqrt{-1} \partial \bar{\partial} \log (-\varphi) \\
& =-\sqrt{-1} \frac{\partial \bar{\partial} \varphi}{\varphi}+\sqrt{-1} \frac{\partial \varphi \wedge \bar{\partial} \varphi}{\varphi^{2}} .
\end{aligned}
$$

Strict plurisubharmonicity of $\varphi$ ensures that $\omega$ is positive. Near the boundary, the volume of $g$ behaves like $O\left((-\varphi)^{-(n+1)}\right)$ (see e.g. Yeg04, Lemma 4.2]), so that $e^{-\psi}=(-\varphi)^{n+2}$ is integrable around $\varphi=0$. By (3.1),$e^{-\lambda(\varphi)} \leqslant e^{-\psi}$, hence $e^{-\lambda(\varphi)}$ is also integrable around $\varphi=0$.

We now prove that the operator $\left(\bar{\partial}+\bar{\partial}_{\lambda(\varphi)}^{*}\right)^{2}$ associated to this metric is Fredholm in bidegree $(0,1)$. We have to show that the estimates (2.1) hold for smooth $(0,1)$ forms which are compactly supported at infinity.

If we are sufficiently far from the boundary $\partial \Omega_{0}$, then the new metric is equal to the original metric of $M$, and the weight $e^{-\lambda(\varphi)}$ is identically one. Therefore, by assumption and by Proposition 2.1, these estimates are valid for forms with compact support in $\Omega_{0}$, sufficiently far from the boundary $\partial \Omega_{0}$.

It remains to prove (2.1) for a $(0,1)$-form $u$ with compact support near the boundary, i.e. in $\{\varphi>-\delta\}$. We shall use the Bochner-Kodaira formula. We endow the trivial line bundle $E=\Omega_{0} \times \mathbb{C}$ with the Hermitian metric $h=e^{-\lambda(\varphi)}$. Consider the holomorphic vector bundle $\widetilde{E}=E \otimes \Lambda^{n} T M$. We denote by $\sim: \Lambda^{0,1} T^{*} M \otimes E \rightarrow$ $\Lambda^{n, 1} T^{*} M \otimes \widetilde{E}, u \mapsto \widetilde{u}$ the natural isometry which sends a $(0,1)$-form with values in $E$ to a $(n, 1)$-form with values in $\widetilde{E}$. 
To avoid excessive subscripts, we will drop $\lambda(\varphi)$ from the notation, and write e.g. $\bar{\partial}^{*}$ instead of $\bar{\partial}_{\lambda(\varphi)}^{*}$. For a $(0,1)$-form $u$ with compact support, we have

$$
\|\bar{\partial} u\|^{2}+\left\|\bar{\partial}^{*} u\right\|^{2}=\|\bar{\partial} \widetilde{u}\|^{2}+\left\|\bar{\partial}^{*} \widetilde{u}\right\|^{2} .
$$

By the Bochner-Kodaira formula for the $(n, 1)$-form $\widetilde{u}$ we have

$$
\|\bar{\partial} \widetilde{u}\|^{2}+\left\|\bar{\partial}^{*} \widetilde{u}\right\|^{2} \geqslant([\sqrt{-1} \Theta(\widetilde{E}), \Lambda] \widetilde{u}, \widetilde{u})
$$

where

$$
\sqrt{-1} \Theta(\widetilde{E})=\sqrt{-1} \Theta(E)+\sqrt{-1} \operatorname{Ric} \omega=\sqrt{-1} \partial \bar{\partial} \lambda(\varphi)+\sqrt{-1} \operatorname{Ric} \omega
$$

is the curvature of $\widetilde{E}$, Ric $\omega$ is the curvature of $\Lambda^{n} T M$ and $\Lambda$ is the interior product with $\omega$. Since $\widetilde{u}$ is of bidegree $(n, 1)$, relations (3.2) and (3.3) imply

$$
\|\bar{\partial} u\|^{2}+\left\|\bar{\partial}^{*} u\right\|^{2} \geqslant(\sqrt{-1} \Theta(\widetilde{E}) \wedge \Lambda \widetilde{u}, \widetilde{u})=(\sqrt{-1}(\partial \bar{\partial} \lambda(\varphi)+\operatorname{Ric} \omega) \wedge \Lambda \widetilde{u}, \widetilde{u}) .
$$

Since $\partial \bar{\partial} \lambda(\varphi)=\lambda^{\prime}(\varphi) \partial \bar{\partial} \varphi+\lambda^{\prime \prime}(\varphi) \partial \varphi \wedge \bar{\partial} \varphi$ we can choose $\lambda$ increasing rapidly enough so that

$$
\sqrt{-1} \partial \bar{\partial} \lambda(\varphi)+\sqrt{-1} \operatorname{Ric} \omega \geqslant \omega
$$

in a neighborhood $\{\varphi>-\delta\}$ of $\partial \Omega_{0}$. Estimates (3.4) and (3.5) entail

$$
\|\bar{\partial} u\|^{2}+\left\|\bar{\partial}^{*} u\right\|^{2} \geqslant(\omega \wedge \Lambda \widetilde{u}, \widetilde{u})=\|\widetilde{u}\|^{2}=\|u\|^{2}
$$

for $u$ supported in $\{\varphi>-\delta\}$. This finishes the proof of the lemma.

The following result is the analog of [MD06, Corollary 2.6]. Note however that we use a complete metric here, which avoids the boundary analysis of [MD06].

Lemma 3.4. Assume that Int $M$ can be endowed with a complete Hermitian metric such that the operator $\left(\bar{\partial}+\bar{\partial}^{*}\right)^{2}$ is Fredholm in bidegree $(0,1)$. Let $p$ be a point of the boundary $\partial \Omega_{0}$, and $f$ a holomorphic function defined in a neighborhood of $p$ such that $\{f=0\} \cap \bar{\Omega}_{0}=\{p\}$. Then for any $m$ big enough, there exist a function $g \in \mathcal{O}\left(\Omega_{0}\right) \cap C^{\infty}\left(\overline{\Omega_{0}} \backslash\{p\}\right)$, a smooth function $\Phi$ on a neighborhood $V$ of $p$, and constants $a_{1}, \ldots, a_{m-1}$ such that

$$
g=\frac{1+a_{m-1} f+\ldots+a_{1} f^{m-1}}{f^{m}}+\Phi
$$

on $V \cap \Omega_{0}$. In particular, we have $\lim _{z \rightarrow p}|g(z)|=\infty$.

Proof. We follow the same line of reasoning as in [MD06, Corollary 2.6] with only minor changes. For $\varepsilon>0$, set

$$
\Omega_{\varepsilon}=\{\varphi<\varepsilon\},
$$

and choose $\varepsilon$ small enough so that $\Omega_{\varepsilon}$ is strongly pseudoconvex. Replacing $\Omega_{0}$ by $\Omega_{\varepsilon}$ in Lemma 3.3. we can find a complete metric $g$ on $\Omega_{\varepsilon}$ and a suitable function $\xi: \Omega_{\varepsilon} \rightarrow \mathbb{R}$, of the form $\xi=\lambda(\varphi-\varepsilon)$, with the properties stated in that lemma. By Proposition 2.2 we have

$$
\operatorname{Ker}\left(\bar{\partial}: L_{2, \xi}^{0,1}\left(\Omega_{\varepsilon}\right) \rightarrow L_{2, \xi}^{0,2}\left(\Omega_{\varepsilon}\right)\right)=\mathscr{H}_{\xi}^{0,1}\left(\Omega_{\varepsilon}, g\right) \oplus \operatorname{Im}\left(\bar{\partial}: L_{2, \xi}^{0,0}\left(\Omega_{\varepsilon}\right) \rightarrow L_{2, \xi}^{0,1}\left(\Omega_{\varepsilon}\right)\right)
$$

and

$$
\operatorname{dim}\left(\mathscr{H}_{\xi}^{0,1}\left(\Omega_{\varepsilon}, \mathrm{g}\right)\right)<\infty .
$$


Now, let $U$ be a small neighborhood of $p$ where $f$ is defined, and let $\psi$ be a smooth function with compact support in $U$, such that $\psi=1$ in a neighborhood $V$ of $p$. Set

$$
h_{m}=\psi / f^{m} \text { on } U \text { and } 0 \text { on } M \backslash U
$$

and

$$
v_{m}=0 \quad \text { on } V \text { and } \bar{\partial} h_{m} \text { on } M \backslash V .
$$

If $\varepsilon$ is sufficiently small, $v_{m}$ is smooth on $\bar{\Omega}_{\varepsilon}$, with compact support (not disjoint from the boundary). In particular, as the weight $e^{-\xi}$ is assumed to be integrable near $\partial \Omega_{\varepsilon}, v_{m}$ is in $L_{2, \xi}^{0,1}\left(\Omega_{\varepsilon}\right)$. Moreover, we have $\bar{\partial} v_{m}=0$ on $\Omega_{\varepsilon}$. By (3.7) and (3.8), the codimension of the image $\operatorname{Im}\left(\bar{\partial}: L_{2, \xi}^{0,0} \rightarrow L_{2, \xi}^{0,1}\right)$ in the kernel $\operatorname{Ker}\left(\bar{\partial}: L_{2, \xi}^{0,1} \rightarrow L_{2, \xi}^{0,2}\right)$ is finite, so that for every $m$ big enough, there are constants $a_{1}, \ldots, a_{m-1}$ such that $v=v_{m}+a_{m-1} v_{m-1}+\ldots+a_{1} v_{1}$ belongs to this image. Hence there is a function $\Phi^{\prime}$ such that $\bar{\partial} \Phi^{\prime}=-v$, and by elliptic regularity, $\Phi^{\prime}$ is smooth on $\Omega_{\varepsilon}$. Set $h=h_{m}+a_{m-1} h_{m-1}+\ldots+a_{1} h_{1}$ and $g=h+\Phi^{\prime}$. Then $\bar{\partial} g=0$ on $\Omega_{\varepsilon} \backslash\{f=0\}$, $g \in \mathcal{O}\left(\Omega_{0}\right) \cap C^{\infty}\left(\bar{\Omega}_{0} \backslash\{p\}\right)$, and the function $\Phi$ in the lemma is equal to $\Phi^{\prime}$ on $V$.

We can now prove Theorem 1.1.

Proof of Theorem 1.1. Let us consider a strictly plurisububharmonic function $\varphi$ as in Consequence 3.2. Let $\varepsilon \in(0, c)$ be sufficiently small. By Lemma 3.4, we can construct peak holomorphic functions for each point of $\partial \Omega_{\varepsilon}=\{\varphi=\varepsilon\}$. Using this fact, it is shown in [MD06, Proposition 3.1] that if $\delta>0$ is sufficiently small, the holomorphic functions on $\{\varphi<\varepsilon\}$ separate points and give local coordinates on $\{\varepsilon-\delta<\varphi<\varepsilon\}$. By the method of [AS70, Proposition 3.2] (see also [MD06, §3]) there exists a compact Stein space $S$ with boundary (and with at most isolated singularities) and an embedding of $\{\varepsilon-\delta<\varphi<\varepsilon\}$ as an open set in $S$ such that $\partial S=\{\varphi=\varepsilon\}$. By gluing $\{\varepsilon-\delta<\varphi<c\}$ with $S$ along $\{\varepsilon-\delta<\varphi<\varepsilon\}$ we obtain a Stein space $S^{\prime}$ with boundary $\{\varphi=c\}$. Using [Heu86, Theorem 0.2] (see also Ohsawa Ohs84) the space $S^{\prime}$ can be embedded as a domain with boundary in a larger Stein space $S^{\prime \prime}$ such that $\partial S^{\prime}$ is a hypersurface in $S^{\prime \prime}$. By the embedding theorem of Remmert-Bishop-Narasimhan Nar60, $S^{\prime \prime}$ admits a proper holomorphic embedding in $\mathbb{C}^{N}$ for some $N$. Restricting this embedding to $\partial S^{\prime}=\{\varphi=c\}=\partial M$ we obtain the conclusion of the theorem.

Remark 3.5. Theorem 1.1 also holds true if we assume that the complete Hermitian metric is defined on $M$ (including the boundary). The same observation applies to Theorem 1.2. However, in the case of quotients in Corollary 1.3 the induced Hermitian metric is defined only in the interior of $M$. (Note that a metric on a manifold with boundary is called complete if the manifold with the induced distance is a complete metric space.)

Remark 3.6. Assume that $M$ is a complex manifold with compact strongly pseudoconvex boundary and complex dimension $n \geq 2$. Assume also that there is a complete Hermitian metric defined on $M$ (or only on its interior) such that for some compact neighborhood $U$ of $\partial M$, we have the Poincaré inequality of Proposition 2.1 for forms of biedegree $(0,1)$ which have compact support in $M \backslash U$. Then this is enough for the conclusion of Lemma 3.3 to hold for $M$ (see the proof). It follows, as in the proof of Theorem 1.1, that $\partial M$ is embeddable. 


\section{CompactificAtion OF MANifolds With PINCHED NEGATIVE CURVATURE}

Let us first of all review some standard facts about manifolds with pinched negative curvature (see the works Eberlein [Ebe80] and Heintze-Im Hof [HIH77], or the book BGS85]). Thus, let $(X, g)$ be any complete manifold with pinched negative sectional curvature $\sigma$, that is, we assume that there are positive constants $a$ and $b$ such that

$$
-b^{2} \leqslant \sigma \leqslant-a^{2}<0 .
$$

For a compact subset $K$ of $X$, an unbounded connected component of $X \backslash K$ is called an end of $X$ (with respect to $K$ ). If $K_{1} \subset K_{2}$ are two compact subsets, the number of ends with respect to $K_{1}$ is at most the number of ends with respect to $K_{2}$, so that we can define the number of ends of $X$. Namely, $X$ is said to have finitely many ends if for some integer $k$, and for any $K \subset X$, the number of ends with respect $K$ is at most $k$. The smallest such $k$ is called the number of ends of $X$, and then there exists $K_{0} \subset X$ such that the number of ends with respect to $K_{0}$ is precisely the number of ends of $X$. If no such $k$ exists, we say that $X$ has infinitely many ends.

Let $E$ be an end of $X$, and assume that $E$ has finite volume. $E$ is called a cusp and is diffeomorphic to a product $(0, \infty) \times \Sigma$ where $\Sigma$ is a connected closed manifold. The slices $\{t\} \times \Sigma$ are the level sets of a Busemann function $r$ associated to $E$. Moreover, the metric restricted to $E$ is given by

$$
\left.g\right|_{E}=d r^{2}+h_{r}
$$

where $h_{r}$ is a family of metrics on $\Sigma$. Finally, standard Jacobi field estimates show that for all $r$, we have $e^{-b r} h_{0} \leqslant h_{r} \leqslant e^{-a r} h_{0}$.

Proof of Theorem 1.2. For the proof of (1), we shall prove that the hypothesis of Theorem 1.1 is fullfilled.

Let $U$ be a neigbourhood of $\partial M$ such that $M \backslash U$ has finite volume. Then $M \backslash U$ has finitely many ends, each of them being a cusp. Using the Busemann functions associated to each of these cusps, it is shown for example in Yeg04, Lemma 3.2] that the Kähler form of the metric is $d$ (bounded) on each cusp. Let us explain the details of the construction. Let $E$ be a cusp of $M$, and let $r$ be a Busemann function associated to $E$ such that $E$ is diffeomorphic to a product $(0, \infty) \times \Sigma$, with $\Sigma$ a closed connected manifold. Let $\varphi_{t}$ be the flow of the gradient $\nabla r$ of $r$. If $x=(s, y)$ is a point of $(0, \infty) \times \Sigma$, we simply have

$$
\varphi_{t}(s, y)=(s+t, y) \text {. }
$$

The properties of the differential of this flow are well understood. First, for $x \in E$, we have $d_{x} \varphi_{t}(\nabla r(x))=\nabla r\left(\varphi_{t}(x)\right)$. Let $u$ be a tangent unitary vector at $x$ and orthogonal to $\nabla r$. Then $t \rightarrow d_{x} \varphi_{t}(u)$ is a stable Jacobi field along the geodesic $t \rightarrow(s+t, y)$, orthogonal to $\nabla r$, and is equal to $u$ at $t=0$. As the sectional curvature is less than or equal to $-a^{2}$, the classical estimates on Jacobi fields HIH77 show that

$$
\left|d_{x} \varphi_{t}(u)\right| \leqslant e^{-a t} .
$$

Now, if $\omega$ is the Kähler form of the metric, it is closed, so that by the Cartan formula, we have

$$
\varphi_{t}^{*} \omega-\omega=d \int_{0}^{t} \varphi_{s}^{*}\left(i_{\nabla r} \omega\right) d s .
$$


By using the estimate of the differential of $\varphi_{t}$, we can take the limit as $t$ goes to infinity and get that $\omega=d \eta$ on the cusp $E$, with

$$
\eta=-\int_{0}^{\infty} \varphi_{s}^{*}\left(i_{\nabla r} \omega\right) d s .
$$

The same estimate implies that $\eta$ is bounded.

From Proposition 2.3 it follows that we have a Poincaré inequality in bidegree $(0,1)$ for forms which have compact support on a cusp. By Theorem 1.1 and Remark 3.6, this achieves the proof of (1).

We consider conclusion (2). The first observation is that $M$ can be compactified, which allows the use of the extension theorem of Kohn-Rossi. Indeed, let $E_{1}, \ldots, E_{m}$ be the cusps of $M \backslash U$. We fix some end $E_{j}$ and consider the associated Busemann function $r: E_{j} \rightarrow(0, \infty)$. It follows from [SY82, Proposition 1] that $-r: E_{j} \rightarrow$ $(-\infty, 0)$ is a strictly plurisubharmonic proper function (note that for the Busemann function, Siu and Yau use the opposite sign convention). From Lemma 3.4 for $\Omega_{0}=\{-r<c\}, c<0$, we infer as in the proof of Theorem 1.1 that each strip $\{c<-r<0\}$ can be compactified to a Stein space with isolated singularities $S_{j, c}$. By the uniqueness of the Stein completion [AS70, Corollary 3.2] all spaces $S_{j, c}$, $c<0$, are biholomorphic to a fixed Stein space $S_{j}$. Letting $c \rightarrow-\infty$, we see that $E_{j}$ is biholomorphic to an open set of $S_{j}$. Therefore all ends of $M \backslash U$, and together with them also $M$, can be compactified.

In particular, the Kohn-Rossi theorem KR65 shows that every holomorphic function defined in a neighborhood of $\partial M$ extends to a holomorphic function on $M$. As a by-product we obtain that $\partial M$ is connected.

Note that, at this stage, by applying [MD06, Theorem 1.3] we can deduce (2) for some strongly pseudoconvex domain $D$, but we cannot say directly that this domain is an open set of a projective manifold. Therefore we proceed as follows. We start by gluing $M$ to a pseudoconcave projective manifold. Since $\partial M$ is embeddable in $\mathbb{C}^{N}$ we can apply [Lem95, Theorem 1.2] which allows us to assume that the Stein space $S^{\prime \prime}$ constructed in the proof of Theorem 1.1 is an open set in an affine algebraic variety, hence also in a projective variety $X$. We now use the notations from the proof of Theorem 1.1. We set $W=\{\varepsilon-\delta<\varphi \leqslant c\}$ and glue the manifolds $M$ and $\left(X \backslash S^{\prime}\right) \cup W$ along $W$. The resulting manifold will be denoted by $\widehat{M}$. Hence $M$ is a domain with compact strongly pseudoconvex boundary in $\widehat{M}$.

Since $S^{\prime \prime}$ is an affine space in some $\mathbb{C}^{N}$, we can regard the embedding of $W^{\prime}=$ $\{\varepsilon-\delta<\varphi<\varepsilon\}$ in $X$ as a map with values in $\mathbb{C}^{N}$. Now $M$ can be compactified to a compact strongly pseudoconvex domain, so the extension theorem of Kohn-Rossi, applied to the components of this embedding, show that the embedding extends to a holomorphic map from $M$ to $\mathbb{C}^{N} \subset \mathbb{P}^{N}$. Pulling back the hyperplane line bundle of $\mathbb{P}^{N}$ through this map, we obtain a line bundle $E \rightarrow \widehat{M}$ which is semi-positive on $\widehat{M}$ and positive on $\left(X \backslash S^{\prime}\right) \cup W$.

A partition of unity argument delivers a complete Hermitian metric $\widehat{\omega}$ on $\widehat{M}$ which agrees with the original metric $\omega$ of Int $M$ on say $\{\varphi<\varepsilon\}$. With respect to the metric induced by $\widehat{\omega}$, the canonical bundle of $M$ is positive on $\{\varphi<\varepsilon\}$. Hence, the bundle $L=E^{k} \otimes K_{\widehat{M}}$ is positive on $\widehat{M}$ for $k$ sufficiently large. Moreover, the curvature $\sqrt{-1} \Theta(L)$ of $L$ dominates $\widehat{\omega}=\omega$ on $\{\varphi<\varepsilon\}$, and therefore dominates $C \widehat{\omega}$ on the whole $\widehat{X}$, for some $C>0$. The metric $\widehat{\omega}$ has bounded torsion and 
Ricci curvature, by construction. Therefore, the $L_{2}$ estimates of Hörmander-SkodaBombieri Dem01] produce sections of $\bigoplus_{\nu} H^{0}\left(\widehat{M}, L^{\nu}\right)$ for $\nu$ sufficiently large, that separate points and give local coordinates on $\widehat{M}$.

On the other hand the manifold $\widehat{M}$ is 1-concave in the sense of Andreotti-Grauert (we again use the plurisuperharmonicity of the Busemann function on each cusp). Therefore, the embedding theorem of Andreotti-Tomassini AT70 implies that the ring $\bigoplus_{\nu} H^{0}\left(\widehat{M}, L^{\nu}\right)$ gives an embedding of $\widehat{M}$ in some projective space $\mathbb{P}^{N}$. By And63 the projective closure of $\widehat{M}$ in $\mathbb{P}^{N}$ is a projective variety $\widetilde{M}$ of the same dimension.

We have thus found a compactification $\widetilde{M} \subset \mathbb{P}^{N}$ of $\widehat{M}$ and therefore of $M$. The desired projective strongly pseudoconvex domain is $D=\widetilde{M} \backslash\left(X \backslash S^{\prime}\right)$. By [Gra62, Satz 3, p.338] there exists a maximal, nowhere discrete analytic set $A$ of $D$ (the exceptional analytic set [Gra62, Definition 3, p. 341]) and by [Gra62, Satz 5, p.340] there exists a Remmert reduction $\pi: D \rightarrow D^{\prime}$, which blows down $A$ to a discrete set of points. The set $D \backslash M=\widetilde{M} \backslash M$ is a pluripolar set, namely the set where the plurisubharmonic function $-r$ takes the value $-\infty$. By the maximum principle for plurisubharmonic functions, $A \subset D \backslash M$. The set $\pi(D \backslash M)$ is also a pluripolar set, and $D_{\text {sing }}^{\prime} \subset \pi(A) \subset \pi(D \backslash M)$. By Wu's theorem [GW79], any simply connected complete Kähler manifold of non-positive sectional curvature is Stein. Hence the universal covering of $M$ is Stein. It is then shown in [SY82, $\S 4]$ (using the SchwarzPick Lemma of Yau) and in [MD06, Theorem 1.3] (using Wermer's theorem) that $D_{\text {sing }}^{\prime}=\pi(D \backslash M)$.

Therefore $D \backslash M=A$ is an exceptional analytic set in the sense of Grauert and by blowing down this exceptional set we obtain the set $D_{\text {sing }}^{\prime}$. Actually, each end $E_{1}, \ldots, E_{m}$ of $M$ can be compactified with one point of the singular set $D_{\text {sing }}^{\prime}=$ $\left\{x_{1}, \ldots, x_{m}\right\}$. Moreover, by the uniqueness of the Stein completion AS70, Cor. 3.2] we see that $D^{\prime}$ and $S^{\prime}$ coincide.

Proof of Corollary 1.3, As is well known, the limit set $\Lambda$ is the set of accumulation points of any orbit $\Gamma \cdot x, x \in B$, and is a closed $\Gamma$-invariant subset of the sphere at infinity $\partial B$. The complement $\partial B \backslash \Lambda$ is precisely the set of points at which $\Gamma$ acts properly discontinuously, and the space $M \cup(\partial B \backslash \Lambda) / \Gamma$ is a manifold with boundary $(\partial B \backslash \Lambda) / \Gamma$ (see for example [EO73, $\S 10]$ ). $A$ is a compact subset of this boundary, hence there is a neighborhood $E$ of $A$ in $M$ which is diffeomorphic to the product $A \times(0,1)$. It follows that $E$ is an end of $M$, because $A$ is compact and connected. Actually, $E$ is a strongly pseudoconvex end, in the sense that its boundary $A$ at infinity is strictly pseudoconvex. Since $M=B / \Gamma$ is a complete manifold with sectional curvature pinched between -4 and -1 , Corollary 1.3 is an immediate consequence of Theorem 1.2 .

Remark 4.1. By [NR98, Theorem 4.2] the following holds:

Theorem (Burns, Napier-Ramachandran). Let $\Gamma$ be a torsion-free discrete group of automorphisms of the unit ball $B$ in $\mathbb{C}^{n}$ with $n \geqslant 3$ and let $M=B / \Gamma$. Assume that the limit set $\Lambda$ is a proper subset of $\partial B$ and that the quotient $(\partial B \backslash \Lambda) / \Gamma$ has a compact component $A$. Then $M$ has only finitely many ends, all of which, except for the unique end corresponding to $A$, are cusps. In fact, $M$ is diffeomorphic to a compact manifold with boundary and can be compactified. 
The proof is based on [NR98, Theorem 4.1] which shows that the finite volume hypothesis of Corollary 1.3 is automatically satisfied in the case $n \geqslant 3$. The presence of the strongly pseudoconvex boundary forces the volume to be finite, since $\partial M$ is then embeddable by BdM75, having real dimension at least 5 .

If $n=2$ we have to assume the volume to be finite in order to obtain the embedding of the boundary. It is interesting to ask whether Burns' theorem also holds in dimension 2 or, equivalently, whether the compact strongly pseudoconvex component of a set $(\partial B \backslash \Lambda) / \Gamma$ is embeddable for all torsion-free discrete groups of automorphisms of the unit ball $B$ in $\mathbb{C}^{2}$.

Let us also remark that the proof of [NR98. Theorem 4.1] also applies in dimension 2 to prove the following converse of Theorem 1.2

Corollary 4.2. Let $M$ be a connected complex manifold with compact strongly pseudoconvex boundary and of complex dimension $n \geqslant 2$. Assume that $M$ is endowed with a complete Kähler metric with pinched negative curvature. The following assertions are equivalent:

(a) $\partial M$ is embeddable in some $\mathbb{C}^{N}$.

(b) $M$ has finite volume away from a neighbourhood of $\partial M$.

Indeed, once $\partial M$ is assumed to be embeddable, we follow the second part of the proof of Theorem 1.2, in order to glue $M$ to a pseudoconcave manifold. To find the bundle $L$ we use the Hartogs phenomenon from [NR98, Proposition 4.4]. Then the holomorphic Morse inequalities yield as in [NR98, Theorem 4.1] that the volume of $M$ is finite away from the pseudoconvex end. Finally, if one of the equivalent conditions (a) or (b) holds true, the argument from Theorem 1.2 shows that $M$ can be compactified to a strongly pseudoconvex domain in a projective variety by adding an exceptional analytic set.

We finish this section by giving an example of a quotient of the unit ball as in Corollary 1.3. We would like to thank T. C. Dinh for communicating to us this example.

Consider the Siegel domain $D \subset \mathbb{C}^{2}$ defined by $\Im w>|z|^{2}$. This domain is biholomorphic to the unit ball $B$ in $\mathbb{C}^{2}$. Consider the group $\Gamma$ of affine automorphisms of $\mathbb{C}^{2}$ generated by the following elements:

$$
\begin{gathered}
S_{a}:(z, w) \mapsto\left(z+a, w+2 i \bar{a} z+b+i|a|^{2}\right), a \in \mathbb{Z}+i \mathbb{Z}, \\
T_{b}:(z, w) \mapsto(z, w+b), b \in \mathbb{Z} .
\end{gathered}
$$

It preserves $D, \partial D$ and the fibration $z=$ const. We can check that $\Gamma$ has no fixed points in the interior of the ball and is discrete.

If $p \in \partial D$, using $S_{a}$ and $T_{b}$ we can prove that the orbit of $p$ contains a point $p_{0}=\left(z_{0}, w_{0}\right)$ such that $\left|z_{0}\right| \leq 2$ and $\left|\Re\left(w_{0}\right)\right| \leq 1$. We also have $\left|\Im\left(w_{0}\right)\right|=\left|z_{0}\right|^{2} \leq 4$. Hence, the action of $\Gamma$ on $\partial D$ is co-compact, and $\bar{D} / \Gamma$ admits a strictly pseudoconvex end.

If $\Gamma_{0}$ denotes the group generated by the $T_{b}$ 's, then $\mathbb{C}^{2} / \Gamma_{0}$ is isomorphic to $\mathbb{C} \times \mathbb{C}^{*}$. The projection $\Pi: \mathbb{C}^{2} \rightarrow \mathbb{C} \times \mathbb{C}^{*}$ is defined by $(z, w) \mapsto(z, \exp (2 i \pi w))$. We can obtain $\mathbb{C}^{2} / \Gamma$ by dividing $\mathbb{C} \times \mathbb{C}^{*}$ by the group $\Gamma^{\prime}$ generated by the following elements:

$$
S_{a}^{\prime}:(z, t) \mapsto\left(z+a, \exp \left(-4 \pi \bar{a} z-2 \pi|a|^{2}\right) t\right) .
$$

The domain $\Pi(D)$ is defined by $|t|<1$. 
We can extend the action of $\Gamma^{\prime}$ to $\mathbb{C} \times \mathbb{C}$ and prove that $S_{a}^{\prime}$ has no fixed point for $a \neq 0$. The group $\Gamma^{\prime}$ preserves the fibration $z=$ const. Hence we can define a map $\pi: \mathbb{C}^{2} / \Gamma^{\prime} \rightarrow T:=\mathbb{C} /(\mathbb{Z}+i \mathbb{Z})$ by $(z, t) \mapsto z$. We can check, using a local coordinate on $T$, that $\pi$ defines a line bundle over $T$.

So $D / \Gamma$ is isomorphic to $\Pi(D) / \Gamma^{\prime}$. Since the zero section of the line bundle has a strongly pseudoconvex neighborhood, it is an exceptional analytic set which can be blown down to a point. Therefore, we can fill up the hyperconcave end of $\Pi(D) / \Gamma^{\prime}$ by adding to $\Pi(D) / \Gamma^{\prime}$ the zero section of the bundle $\pi: \mathbb{C}^{2} / \Gamma^{\prime} \rightarrow T$.

\section{EMBEDding OF SASAKIAN 3-MANifoldS}

5.1. Sasakian manifolds. In this section, we explain some well known facts about $\mathrm{CR}$ and Sasakian manifolds. Let $Y$ be a smooth orientable manifold of (real) dimension $(2 n-1)$. A $C R$ structure on $Y$ is an $(n-1)$-dimensional complex subbundle $H_{(1,0)} Y$ of the complexified tangent bundle $T_{\mathbb{C}} Y$ such that

$$
H_{(1,0)} Y \cap \overline{H_{(1,0)} Y}=\{0\},
$$

and such that $H_{(1,0)}$ is integrable as a complex subbundle of $T_{\mathbb{C}} Y$ (i.e. if $u$ and $v$ are sections of $H_{(1,0)} Y$, the Lie bracket $[u, v]$ is still a section of $\left.H_{(1,0)} Y\right)$.

If $Y$ is a CR manifold, then its Levi distribution $H$ is the real subbundle of $T Y$ defined by $H=\operatorname{Re}\left\{H_{(1,0)} Y \oplus \overline{H_{(1,0)} Y}\right\}$. There exists on $H$ a complex structure $J$ given by $J(u+\bar{u})=\sqrt{-1}(u-\bar{u})$, with $u \in H_{(1,0)} Y$. As $Y$ is orientable, the real line bundle $H^{\perp} \subset T^{*} Y$ admits a global non-vanishing section $\theta$. The CR structure is said to be strongly pseudoconvex if $d \theta(., J$.$) defines a positive definite metric on$ $H$. Notice that in this case, $\theta \wedge(d \theta)^{n-1} \neq 0$, and $\theta$ defines a real contact structure on $Y$. Let $X$ be a strictly pseudoconvex $C R$ manifold, with compatible complex structure $J$, and compactible contact form $\theta$. This allows us to define a Riemannian metric $g_{\theta}$ on $X$ given by

$$
g_{\theta}(., .)=d \theta(., J .)+\theta(.) \theta(.) .
$$

Let $R$ be the Reeb vector field associated to $\theta$, defined by

$$
i_{R} \theta=1, \quad i_{R} d \theta=0 .
$$

Associated to the data $\left(X, \theta, R, J, g_{\theta}\right)$, there is a canonical connection $\nabla$ on $T X$, called the Tanaka-Webster connection (see Tanaka Tan75] and Webster Web78]), which is the unique affine connection on $T X$ such that

(1) $\nabla g_{\theta}=0, \nabla J=0, \nabla \theta=0$.

(2) For any $u, v$ in the Levi distribution $H$, the torsion $T$ of $\nabla$ satisfies $T(u, v)=$ $d \theta(u, v) R$ and $T(R, J u)=J T(R, u)$.

In particular, the torsion of the Tanaka-Webster connection cannot vanish identically. However, we have the following definition.

Definition 5.1. A strictly pseudoconvex manifold is called a Sasakian manifold if the torsion of its Webster connection in the direction of the Reeb vector field vanishes, i.e. $T(R,)=$.0 with the notation above.

Examples of compact Sasakian manifolds are the unit sphere in $\mathbb{C}^{n}$ or the Heisenberg nilmanifold (see Urakawa Ura93]). Sasakian 3-manifolds were classified up to diffeomorphism by H. Geiges Gei97. They were further studied by F. Belgun in Bel01: every Sasakian 3-manifold is obtained as a deformation of some standard model (see Bel01] for more details). 
5.2. Asymptotically complex hyperbolic manifolds. In [BH02, O. Biquard and M. Herzlich consider a class of manifolds which are modelled on the complex unit ball, and are thus called asymptotically complex hyperbolic. This construction will allow us to get an embedding theorem for Sasakian manifolds, but let us first recall the meaning of an asymptotically complex hyperbolic manifold.

Let $X$ be a $(2 m-1)$-dimensional compact manifold, $m \geqslant 2$. We assume that $X$ has a strongly pseudoconvex CR-structure. Let $\theta$ be a compatible contact form, and $J$ a compatible almost complex structure. $\gamma(.,):.=d \theta(., J$.$) is then a metric$ on the contact distribution. Following Biquard and Herzlich BH02, we endow $\Omega:=(0, \infty) \times X$ with the metric

$$
g=d r^{2}+e^{-2 r} \theta^{2}+e^{-r} \gamma .
$$

Actually, in $\mathrm{BH} 02$, the authors consider the metric $d r^{2}+e^{2 r} \theta^{2}+e^{r} \gamma$ on $\Omega$, but the reason for our choice will become clear later.

We can extend the almost complex structure to act on the whole tangent bundle as follows. Consider the Reeb vector field $R$ and define

$$
J \partial_{r}=e^{r} R,
$$

where $\partial_{r}$ is the unit vector field in the $r$ direction. $g$ is then a Hermitian metric with respect to $J$, i.e. $J$ is an $g$-isometry. The fundamental 2 -form associated to $g$ is

$$
\omega=d\left(e^{-r} \theta\right)
$$

Although $\omega$ is a closed form, in this general setting, $((0, \infty) \times X, g)$ is not necessarily a Kähler manifold, because $J$ is not necessarily an integrable almost complex structure. Indeed, the proof of [BH02, Proposition 3.1] shows that $J$ is integrable if and only if the torsion of the Webster connection of $(X, \theta)$ in the direction of the Reeb vector field vanishes identically, i.e. if and only if $X$ is a Sasakian manifold (see Definition 5.1).

From now on, $X$ will be a Sasakian manifold, so that $(\Omega, g)$ is a Kähler manifold. Note that $\eta:=e^{-r} \theta$ is a bounded $1-$ form, hence the Kähler form (5.2) associated to $g$ is $d$ (bounded). In particular, by Proposition 2.3 , the operator $\left(\bar{\partial}+\bar{\partial}^{*}\right)^{2}$ on $(\Omega, g)$ is Fredholm in bidegree $(0,1)$ if $\operatorname{dim}_{\mathbb{R}} X \geqslant 3$. Moreover, we can also write

$$
\omega=-2 \sqrt{-1} \partial \bar{\partial} r
$$

so that $\Omega$ has a stromgly pseudoconvex boundary $\{0\} \times X$ (this explains the choice of sign of the $r$ variable for $g$ in (5.1): with our choice, $\Omega$ has a strongly pseudoconvex boundary, whereas with the choice of $[\mathrm{BH} 02] \Omega$ has a strongly pseudoconcave boundary). From Theorem 1.1 we can conclude:

Theorem 5.2. Let $X$ be a Sasakian manifold of dimension at least 3 . Then there is a $C R$ embedding of $X$ in $\mathbb{C}^{N}$ for some integer $N$.

Remark. The function $-r: \Omega \rightarrow(-\infty, 0)$ is a proper smooth strictly plurisubharmonic function. Therefore $\Omega$ is a hyperconcave end, and our theorem is also a direct consequence of MD06].

\section{ACKNOWLEDGEMENTS}

We would like to thank G. Carron, T. C. Dinh and M. Herzlich for their interest in this work. Moreover, the second-named author thanks J. Brüning and the 
"Geometric Analysis and Spectral Theory" group of the Humboldt Universität zu Berlin for their hospitality.

\section{REFERENCES}

[And63] A. Andreotti, Théorèmes de dépendance algébrique sur les espaces complexes pseudoconcaves, Bull. Soc. Math. France 91 (1963), 1-38. MR0152674 (27:2649)

[Ang93] N. Anghel, An abstract index theorem on noncompact Riemannian manifolds, Houston J. Math. 19 (1993), no. 2, 223-237. MR.1225459 (94c:58193)

[AS70] A. Andreotti and Y.-T. Siu, Projective embedding of pseudoconcave spaces, Ann. Scuola Norm. Sup. Pisa (3) 24 (1970), 231-278. MR0265633 (42:542)

[AT70] A. Andreotti and G. Tomassini, Some remarks on pseudoconcave manifolds, Essays on Topology and Related Topics (Mémoires dédiés à Georges de Rham), Springer, New York, 1970, pp. 85-104. MR0265632 (42:541)

[BGS85] W. Ballmann, M. Gromov, and V. Schroeder, Manifolds of nonpositive curvature, Progress in Mathematics, vol. 61, Birkhäuser Boston, Inc., Boston, MA, 1985. MR0823981 (87h:53050)

[Bel01] F. Belgun, Normal CR structures on compact 3-manifolds, Math. Z. 238 (2001), no. 3, 441-460. MR.1869692 (2002k:32065)

[BH02] O. Biquard and M. Herzlich, A Burns-Epstein invariant for ACHE 4-manifolds, Duke Math. J. 126 (2005), no. 1, 53-100. MR2110628 (2006g:32034)

[BE96] J. Bland and C. L. Epstein, Embeddable CR-structures and deformations of pseudoconvex surfaces. I. Formal deformations, J. Algebraic Geom. 5 (1996), no. 2, 277-368. MR.1374711 (97c:32021)

[BdM75] L. Boutet de Monvel, Intégration des équations de Cauchy-Riemann induites formelles, Séminaire Goulaouic-Lions-Schwartz 1974-1975; Équations aux derivées partielles linéaires et non linéaires, Centre Math., École Polytech., Paris, 1975, pp. Exp. No. 9, 14. MR0409893 (53:13645)

[BE90] D. M. Burns and C. L. Epstein, Embeddability for three-dimensional CR-manifolds, J. Amer. Math. Soc. 3 (1990), no. 4, 809-841. MR1071115 (93b:32024)

[Bur79] D. M. Burns, Global behavior of some tangential Cauchy-Riemann equations, Partial differential equations and geometry (Proc. Conf., Park City, Utah, 1977), Lecture Notes in Pure and Appl. Math., vol. 48, Dekker, New York, 1979, pp. 51-56. MR0535588 (81d:32032)

[Cat] D. Catlin, A Newlander-Nirenberg theorem for manifolds with boundary, Michigan Math. J., 35 (1988), no. 2, 233-240. MR0959270 (89j:32026)

[Dem01] J.-P. Demailly, Complex analytic and differential geometry, published online at wwwfourier.ujf-grenoble.fr/ ${ }^{\sim}$ demailly/lectures.html, 2001.

[Ebe80] P. Eberlein, Lattices in spaces of nonpositive curvature, Ann. of Math. (2) 111 (1980), no. 3, 435-476. MR0577132 (82m:53040)

[EO73] P. Eberlein and B. O'Neill, Visibility manifolds, Pacific J. Math. 46 (1973), 45-109. MR0336648 (49:1421)

[EH00] C. L. Epstein and G. M. Henkin, Stability of embeddings for pseudoconcave surfaces and their boundaries, Acta Math. 185 (2000), no. 2, 161-237. MR1819994 (2002e:32048)

[Fa192] E. Falbel, Nonembeddable CR-manifolds and surface singularities, Invent. Math. 108 (1992), no. 1, 49-65. MR1156386 (93j:32016)

[Gei97] H. Geiges, Normal contact structures on 3-manifolds, Tohoku Math. J. (2) 49 (1997), no. 3, 415-422. MR.1464186 (98h:53046)

[Gra62] H. Grauert, Über Modifikationen und exzeptionelle analytische Mengen, Math. Ann. 146 (1962), 331-368. MR0137127 (25:583)

[GW79] R. E. Greene and H. Wu, Function theory on manifolds which possess a pole, Lecture Notes in Mathematics, vol. 699, Springer, Berlin, 1979. MR0521983 (81a:53002)

[Gro91] M. Gromov, Kähler hyperbolicity and $L_{2}$-Hodge theory, J. Differential Geom. 33 (1991), no. 1, 263-292. MR.1085144 (92a:58133)

[HL75] F. R. Harvey and H. Blaine Lawson, Jr., On boundaries of complex analytic varieties. I, Ann. of Math. (2) 102 (1975), no. 2, 223-290. MR0425173 (54:13130)

[HIH77] E. Heintze and H.-C. Im Hof, Geometry of horospheres, J. Differential Geom. 12 (1977), no. 4, 481-491 (1978). MR0512919(80a:53051) 
[Heu86] D. Heunemann, Extension of the complex structure from Stein manifolds with strictly pseudoconvex boundary, Math. Nachr. 128 (1986), 57-64. MR0855943 (87j:32057)

[KR65] J. J. Kohn and H. Rossi, On the extension of holomorphic functions from the boundary of a complex manifold, Ann. of Math. (2) 81 (1965), 451-472. MR0177135 (31:1399)

[Lem95] L. Lempert, Algebraic approximations in analytic geometry, Invent. Math. 121 (1995), no. 2, 335-353. MR:1346210 (97k:32021)

[MD06] G. Marinescu and T-C. Dinh, On the compactification of hyperconcave ends and the theorems of Siu-Yau and Nadel, Invent. Math. 164 (2006), no. 2, 233-248. MR2218480 (2007b:32033b)

[NT88] A. Nadel and H. Tsuji, Compactification of complete Kähler manifolds of negative Ricci curvature, J. Differential Geom. 28 (1988), no. 3, 503-512. MR0965227 (89m:32047)

[NR98] T. Napier and M. Ramachandran, The $L^{2} \bar{\partial}$-method, weak Lefschetz theorems, and the topology of Kähler manifolds, J. Amer. Math. Soc. 11 (1998), no. 2, 375-396. MR 1477601 (99a:32008)

[Nar60] R. Narasimhan, Imbedding of holomorphically complete complex spaces, Amer. J. Math. 82 (1960), 917-934. MR0148942 (26:6438)

[Ohs84] T. Ohsawa, Holomorphic embedding of compact s.p.c. manifolds into complex manifolds as real hypersurfaces, Differential geometry of submanifolds (Kyoto, 1984), Lecture Notes in Math., vol. 1090, Springer, Berlin, 1984, pp. 64-76. MR.0775145 (86j:32047)

[Ros65] H. Rossi, Attaching analytic spaces to an analytic space along a pseudoconcave boundary, Proc. Conf. Complex Analysis (Minneapolis, 1964), Springer, Berlin, 1965, pp. 242-256. MR0176106 (31:381)

[SY82] Y. T. Siu and S. T. Yau, Compactification of negatively curved complete Kähler manifolds of finite volume, Seminar on Differential Geometry, Ann. of Math. Stud., vol. 102, Princeton Univ. Press, Princeton, N.J., 1982, pp. 363-380. MR0645748 (83g:32027)

[Tan75] N. Tanaka, A differential geometric study on strongly pseudo-convex manifolds, Kinokuniya Book-Store Co. Ltd., Tokyo, 1975, Lectures in Mathematics, Department of Mathematics, Kyoto University, No. 9. MR0399517 (53:3361)

[Ura93] H. Urakawa, Variational problems over strongly pseudoconvex CR manifolds, Differential geometry (Shanghai, 1991), World Sci. Publishing, River Edge, NJ, 1993, pp. 233-242. MR1341616(96h:58048)

[Web78] S. M. Webster, Pseudo-Hermitian structures on a real hypersurface, J. Differential Geom. 13 (1978), no. 1, 25-41. MR0520599 (80e:32015)

[Yeg04] N. Yeganefar, $L^{2}$-cohomology of negatively curved Kähler manifolds of finite volume, to appear in Geom. and Funct. Analysis, arXiv:math.DG/0402056, 2004.

Institut für Mathematik, Humboldt-Universität Zu Berlin, Unter den Linden 6, 10099 Berlin, Germany

E-mail address: george@mathematik.hu-berlin.de

Current address: Mathematisches Institut, Universität zu Köln, Weyertal 86-90, D50931 Köln, Germany

E-mail address: gmarines@math.uni-koeln.de

Département de Mathématiques, Université de Nantes, 2 rue de la Houssinière, BP 92208, 44322 Nantes Cedex 03, France

E-mail address: nader.yeganefar@math.univ-nantes.fr

Current address: CMI, Université de Provence, 39 Rue Frédéric Joliot Curie, 13453 Marseille cedex 13, France

E-mail address: Nader.Yeganefar@cmi.univ-mrs.fr 